\title{
Noise Properties and Time Response of the Staircase Avalanche Photodiode
}

\author{
KUNIAKI MATSUO, MALVIN C. TEICH, SENIOR MEMBER, IEEE, \\ AND BAHAA E. A. SALEH, MEMBER, IEEE
}

\begin{abstract}
$A b s t r a c t-T h e$ staircase avalanche photodiode is a novel graded-gap superlattice device that is expected to detect photons quite noiselessly. It is designed in such a way that only electrons impact-ionize, thereby eliminating the feedback noise associated with conventional two-carrier avalanche devices. Because the electron multiplication can occur only at a small number of discrete locations in the device, the variability of the number of electrons generated per detected photon is minimized. The excess noise and the electron counting distribution are obtained as a function of the number of stages in the device and the impact-ionization probability per stage, for instantaneous multiplication. The (single-photon) impulse response function is calculated when the effects of (random) transit time are incorporated into the carrier multiplication process. Inclusion of the time dynamics is essential for determining the time course of the current generated by the device in response to pulses of light. This, in turn, permits bit error rates to be calculated for systems incorporating the device. For a five-stage quaternary device the gain-bandwidth product is calculated to be in the vicinity of $600 \mathrm{GHz}$.
\end{abstract}

\section{INTRODUCTION}

\section{A. Background}

A VALANCHE photodiodes (APD's) are important devices, in large part because of their use as detectors in fiber-optic communication systems [1]. They operate by converting each detected photon into a cascade of electrons; a pulse of light then produces a sufficiently high charge to be readily detected by the electronics following the APD. This multiplication (or branching) process introduces noise because the number of electrons created per detected photon varies from trial to trial. Nevertheless, this noise is tolerated because it is usually less detrimental to system performance than is the additive noise of the amplifiers and other electronics following the APD.

The performance of an optical communication system using conventional single- and double-carrier APD's has been studied in detail by McIntyre [2]-[4] and Personick [5], [6] for the case where the carriers multiply instantaneously. The time dynamics of the single-carrier multiplication process can be incorporated into the theory by considering avalanching in terms of a Poisson branching

Manuscript received May 20, 1985; revised August 14, 1985. This research was supported by the National Science Foundation and by the Joint Services Electronics Program (U.S. Army, U.S. Navy, and U.S. Air Force) under contract DAAG29-85-K-0049.

K. Matsuo and M. C. Teich are with the Columbia Radiation Laboratory, Department of Electrical Engineering, Columbia University, New York, NY 10027.

B. E. A. Saleh is with the Department of Electrical and Computer Engineering, University of Wisconsin, Madison, WI 53706. point process [7]. From this vantage point, it is apparent that the simplest case treated by McIntyre and Personick (single-carrier injection, single-carrier instantaneous multiplication) is identical to the well-known Yule-Furry branching process [8], [9].

Single-carrier multiplication is desirable because it is substantially less noisy than double-carrier multiplication [4], [5]. However, at the longer wavelengths currently of interest for fiber-optic communications, single-carrier multiplication is difficult to achieve in conventional III-V devices because the electrons and holes generally have ionization coefficients that are similar [10]. Another problem at these longer wavelengths is high tunneling currents (which increase with increasing reverse bias). As a result, there have recently been proposals for a number of novel heterostructure devices that reduce tunneling currents and enhance the ionization-coefficient ratio (i.e., render the multiplication more single-carrier-like) [10]. One ingeneous proposal, by Chin et al. [11], suggested the use of a superlattice of alternating wide- and narrow-bandgap layers. A device of this type was recently fabricated by Capasso et al. [12]; indeed, an enhancement of the ionization-rate ratio was evident.

In this paper, we calculate the noise properties and single-photon impulse response function for a new type of APD whose behavior is predicted to be substantially quieter than that of the conventional device. In a recent set of theoretical papers, Williams, Capasso, and Tsang [13], [14] proposed a staircase solid-state avalanche photodiode (SAPD) with an enhanced ionization ratio that is predicted to detect light quite noiselessly. The device makes use of a graded-gap superlattice structure [15]. It is designed so that only electrons impact-ionize, thereby eliminating the feedback noise associated with two-carrier devices. Furthermore, because the electron multiplication occurs only at discrete locations in the device, the variability of the number of electrons generated per photon is reduced. These two factors promise a low-noise device that is potentially attractive for use in fiber-optic communication systems and in other configurations that demand ultralownoise performance. The device will operate at low voltages, thereby minimizing leakage currents.

\section{B. Description of the Device}

The SAPD is a multilayer structure in which the ionization energy is provided exclusively by conduction-band 


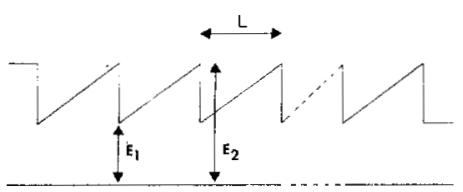

(a)

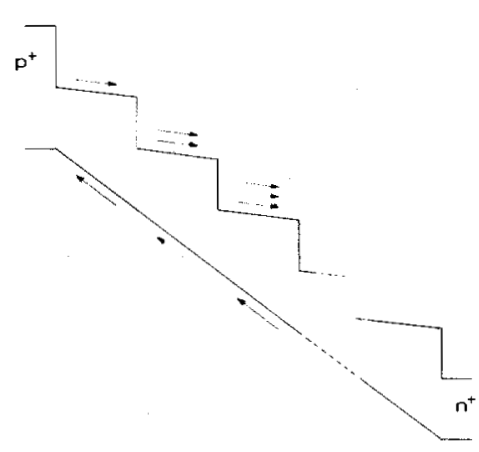

(b)

Fig. 1. Energy-band diagram of the staircase avalaniche photodiode (SAPD) under (a) unbiased and (b) biased conditions. Valence-band steps are assumed to be negligible; the flow of arrows in the valence band signifies that holes do not impact-ionize.

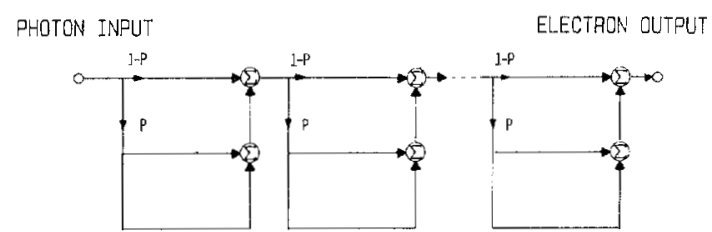

Fig. 2. Particle-flow diagram for the SAPD with instantaneous multiplication. Photon input signifies an effective photon absorption in the device. $P$ is the impact-ionization probability. Three stages are explicitly illustrated; each is a shifted Bernoulli distribution.

discontinuities [13]-[15]. The energy-band diagram of the device at zero applied field is illustrated in Fig. 1(a). Each stage is linearly graded in composition from a low $\left(E_{1}\right)$ to a high $\left(E_{2}\right)$ bandgap, with an abrupt step back to the low bandgap. The diagram is idealized in that there are assumed to be no valence-band steps; thus only electrons are assumed to impact-ionize. Under biased conditions, the energy-band diagram takes the form illustrated in Fig. 1(b), whence the name "staircase avalanche photodiode." Electron impact ionizations are permitted only at the abrupt steps in the staircase.

Ideally, each electron gives rise to a daughter electronhole pair (with probability $P=1$ ) at the step. The total number of electronic charges induced in the external circuit is then $2^{m}$, in which case the multiplication is deterministic and the device noiseless. In practice, the impactionization probability $P$ lies below unity and multiplication noise is present. A particle-flow diagram representing the process of carrier multiplication is presented in Fig. 2 for an $m$-stage device (three stages are explicitly shown). Because this diagram represents instantaneous multiplication it suffices to consider only electrons. A single photon absorbed at the input of the device generates a primary electron. At the output of the first stage, the initial electron either produces a daughter (with probability $P$ ) re- sulting in two electrons, or fails to produce a daughter (with probability $1-P$ ) in which case the primary electron remains and several phonons are produced. Each electron at the output of the first stage independently goes through the same process at the $m-1$ subsequent stages.

\section{Excess Noise Factor and Probability of Error}

A simple measure of the avalanche noise in an instantaneous-multiplication APD is provided by the excess noise factor $F_{e}$. This quantity is the second moment of the gain (or multiplication) random variable for a single event at the input $\left\langle N_{m}^{2}\right\rangle$, normalized by the square of the mean gain $\left\langle N_{m}\right\rangle^{2}$

$$
F_{e}=\left\langle N_{m}^{2}\right\rangle /\left\langle N_{m}\right\rangle^{2}
$$

The excess noise factor can be expressed in terms of the mean and variance $\left(\operatorname{var}\left(N_{m}\right)\right)$ of the electron count that results from a single primary event by the relation

$$
F_{e}=1+\left[\operatorname{var}\left(N_{m}\right) /\left\langle N_{m}\right\rangle^{2}\right] \text {. }
$$

For deterministic multiplication (i.e., the absence of carrier fluctuations), $\operatorname{var}\left(N_{m}\right)=0$ and $F_{e}=1$. From this point of view, the expected noise reduction provided by the SAPD is substantial: $F_{e}$ for conventional APD's usually exceeds 2 and, depending on the device material and structure, can rise one or two orders of magnitude above this; $F_{e}$ for the single-carrier SAPD always lies below 2 .

The excess noise factor and the electron count variance are useful statistics because they represent, in a compact way, the lowest order statistical properties of the gain fluctuations that impose multiplicative noise on individual photon detections (excess noise). However, it is clear that these quantities provide an incomplete statistical description of the device output. Although they are useful for calculating quantities such as the conventional signal-to-noise ratio (SNR) for analog detection, they are inadequate for calculating the performance of a digital-signal information transmission system [16]. The latter is characterized by measures such as probability of detection and probability of error, which depend on a more complete statistical description of the output (beyond the second moment). For an integrate-and-dump receiver, the quantity of interest is the charge induced in the external circuit. For this purpose we require the probability distribution of the number of electrons, or counting distribution, as it is often called.

\section{Approach}

We consider a cascade of shifted Bernoulli distributions, each of which takes on two possible values: 2 with probability $P$, and 1 with probability $1-P$. In the context of a branching process [7], [17], we calculate the moment generating function, moments, and counting distribution for the electrons produced at the output of the SAPD when the multiplication is instantaneous. This is illustrated in Fig. 2. We then proceed to incorporate the effects of time dynamics into the multiplication process in order to examine the time course of the output current (single-photon impulse response function). This is carried out in two 
stages. First, a model incorporating deterministic transit time is formulated and then, in a closer approximation to reality, the transit time is permitted to become random. The impulse-response function determines how quickly successive bits can be sent with minimal interference and permits system performance to be evaluated. With the enormous increases in bit rate that have recently been made available by fast optical pulse generation and transmission techniques, the speed of response of the APD is a critical factor in determining limits for the informationcarrying capacity of optical communication systems.

\section{iI. Instantaneous Multiplication Statistics}

We evaluate the statistical properties of the electrons at the output of the SAPD when the multiplication is instantaneous. These results also apply when time dynamics are incorporated in the multiplication but the integration time at the output of the device exceeds the duration of the impulse response function. The properties of interest are the moment-generation function, moments (particularly the excess noise factor), and counting distribution. Actually, the excess noise factor for the staircase device is properly described by the mathematical model set forth by van Vliet and Rucker [18], [19]. Although their treatment was intended to describe multiplication noise in conventional APD's, their use of a deterministic number of shifted-Bernoulli stages makes their model suitable for the instantaneous-multiplication staircase device instead. They did not calculate the counting distribution for a finite number of stages, however.

\section{A. Moment Generating Function}

The random variable of interest is the total number of electrons $N_{m}$ generated at the output of the $m$-stage device. The moment generating function $(m g f)$ is defined by the expression

$$
Q_{m}(s)=\left\langle\exp \left(-s N_{m}\right)\right\rangle
$$

where the expectation is understood to be over the probability distribution of $N_{m}$. The $m g f$ of the Bernoulli branching process is obtained as a special case of the Galton-Watson branching process [17]. It is expressed quite directly in terms of the nested relationship [7]

$$
\begin{aligned}
& Q_{m}(s)=(1-P) Q_{m-1}(s)+P\left[Q_{m-1}(s)\right]^{2}, \quad m \geq 1 \\
& Q_{0}(s)=e^{-s} .
\end{aligned}
$$

The moments of the electron number are obtained from (4).

\section{B. Moments and Excess Noise Factor}

From Fig. 2 it is readily apparent that the mean electron count has the value

$$
\left\langle N_{m}\right\rangle=(1+P)^{m}, \quad m \geq 1
$$

which reduces to $2^{m}$ in the case of ideal multiplication, as indicated earlier. Equation (5) can, of course, also be obtained directly from the moment generating function in (4) by using the well-known relation [20]

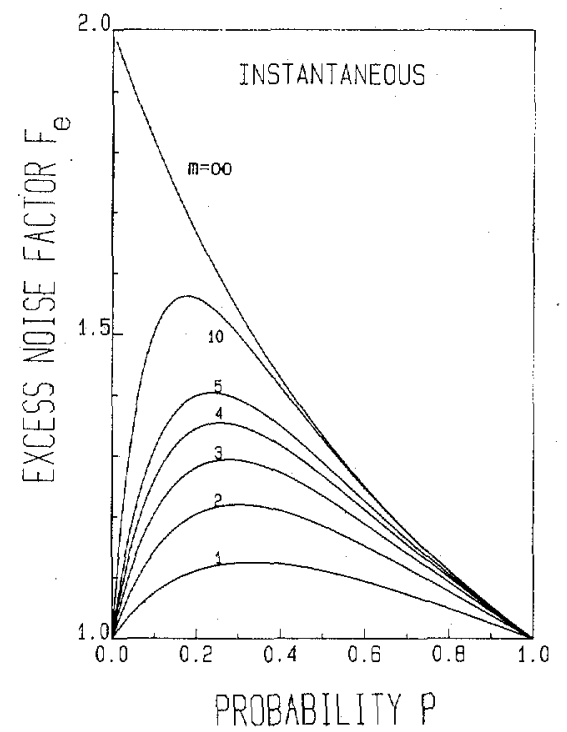

Fig. 3. Excess noise factor $F_{e}$ versus impact-ionization probability $P$ for instantaneous-multiplication staircase avalanche photodiode. The number of stages is represented by the parameter $m$. For $P>0.83, F_{c}<$ 1.1 for all $m$.

$$
\left\langle N_{m}\right\rangle=-\left.\frac{\partial}{\partial s} Q_{m}(s)\right|_{s=0} .
$$

Similarly, the second moment is given by [20]

$$
\begin{aligned}
\left\langle N_{m}^{2}\right\rangle= & \left.\frac{\partial^{2}}{\partial s^{2}} Q_{m}(s)\right|_{s=0}=(1+3 P)(1+P)^{m-1} \\
& +2(1+P)^{m}\left[(1+P)^{m-1}-1\right] .
\end{aligned}
$$

From (1) the excess noise factor is calculated to be

$$
F_{e}=1+[(1-P) /(1+P)]\left[1-(1+P)^{-m}\right] \text {. }
$$

Rewriting this as a function of the mean count $\left\langle N_{m}\right\rangle$ provides

$$
F_{e}=\left\langle N_{m}\right\rangle^{-1}+2\left\langle N_{m}\right\rangle^{-1 / m}-2\left\langle N_{m}\right\rangle^{-1-1 / m} .
$$

Finally, taking (8b) to the limit $m \rightarrow \infty$ leads to

$$
F_{e m \rightarrow \infty}=\begin{array}{ll}
1, & P=0 \\
2 /(1+P), & 0<P<1
\end{array}
$$

illustrating that the excess noise factor cannot exceed 2.

A graphical representation of the excess noise factor in (8a), as a function of $P$ and $m$, is provided in Fig. 3. This result is equivalent to that provided by van Vliet and Rucker, who computed expressions for the count mean and variance [18, eqs. (40) and (55)]; the conversion is effected by using (2). Employing a different rationale, $\mathrm{Ca}$ passo et al. [14, eq. (2), fig. 2] calculated the excess noise factor for the SAPD and arrived at a result that is identical to that provided in (8a) and Fig. 3.

A close examination of Fig. 3 illustrates that $F_{e}=1$ when $P=0,1$, independent of $m$. At these two extremes the multiplication is deterministic and the device introduces no noise. Also, for any fixed $m$ there is a least desirable value of $P$ at which the excess noise factor is max- 
imum. Furthermore, for any fixed $P$, increasing $m$ is accompanied by increasing $F_{e}$. For every $m$, as $P$ moves toward unity, the excess noise factor becomes smaller and the gain increases, indicating that $P$ should be made as large as possible.

The introduction of time dynamics into the calculations has no effect on the excess noise factor, or on the counting distribution, as long as the integration time is sufficiently long so that the entire current pulse is captured as a charge. Although the effect of transit time is to produce a continuous current in the circuit, it does not alter the total number of electrons generated per detected photon.

\section{Counting Distribution}

The probability generating function $(p g f)$ for the $m$ stage case is defined by the expression

$$
G_{m}(z)=\left\langle z^{N_{m}}\right\rangle .
$$

The $p g f$ is easily obtained from the moment generating function provided in (4) by effecting the substitution $\exp (-s) \rightarrow z[20]$ whereupon

$$
\begin{aligned}
& G_{m}(z)=(1-P) G_{m-1}(z)+P\left[G_{m-1}(z)\right]^{2}, \quad m \geq 1 \\
& G_{0}(z)=z .
\end{aligned}
$$

This result is appropriate for a single primary event.

The electron counting distribution is obtained from the $p g f$ by means of the formula [20]

$$
p_{m}(n)=\left.\frac{1}{n !} \frac{\partial^{n}}{\partial z^{n}} G_{m}(z)\right|_{z=0} .
$$

A straightforward calculation using (10) and (11) yields

$$
\begin{aligned}
& p_{m}(n)=(1-P) p_{m-1}(n) \\
& \quad+P \sum_{k=0}^{n} p_{m-1}(n-k) p_{m-1}(k), \quad n \geq 1, \quad m \geq 1
\end{aligned}
$$

$p_{m}(0)=0, \quad m \geq 1$

$P_{0}(n)=\delta_{1, n} . \quad m \geq 1$

In Fig. 4 we plot the counting distributions $p_{3}(n)$ versus $n(m=3)$ for $P=0.2,0.8$, and 0.95 . Higher count numbers achieve greater probabilities as $P$ increases, with a maximum count of $2^{3}=8$. The spread in the distribution (representing the excess noise factor) becomes quite small as $P$ approaches unity, in accord with the behavior evident in Fig. 3. In Fig. 5, we present the counting distributions $p_{10}(n)$ versus $n$, for the same three values of $P$. The behavior is similar; as $P$ increases the distribution increases in mean value and decreases in width. The maximum permitted count in this case is $2^{10}=1024$ and the average gain is $(1.95)^{10} \approx 800$ for $P=0.95$.

An interesting feature of the multiplication process is evident in the multiple peaks of the counting distributions. These result from failures to multiply early in the chain. Consider an unlucky electron that does not manage to

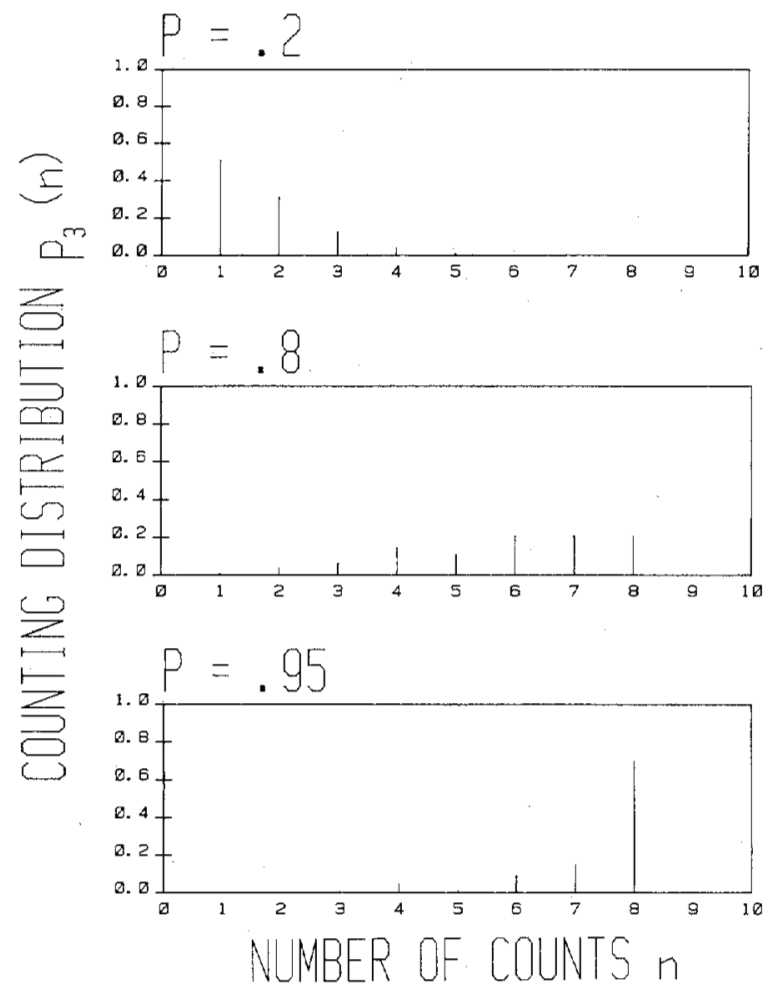

Fig. 4. Electron counting distribution $p_{3}(n)$ versus $n$, for $m=3$, with ionization probability $P=0.2$ (top), $P=0.8$ (middle), and $P=0.95$ (bottom). The maximum multiplication is $2^{3}=8$.
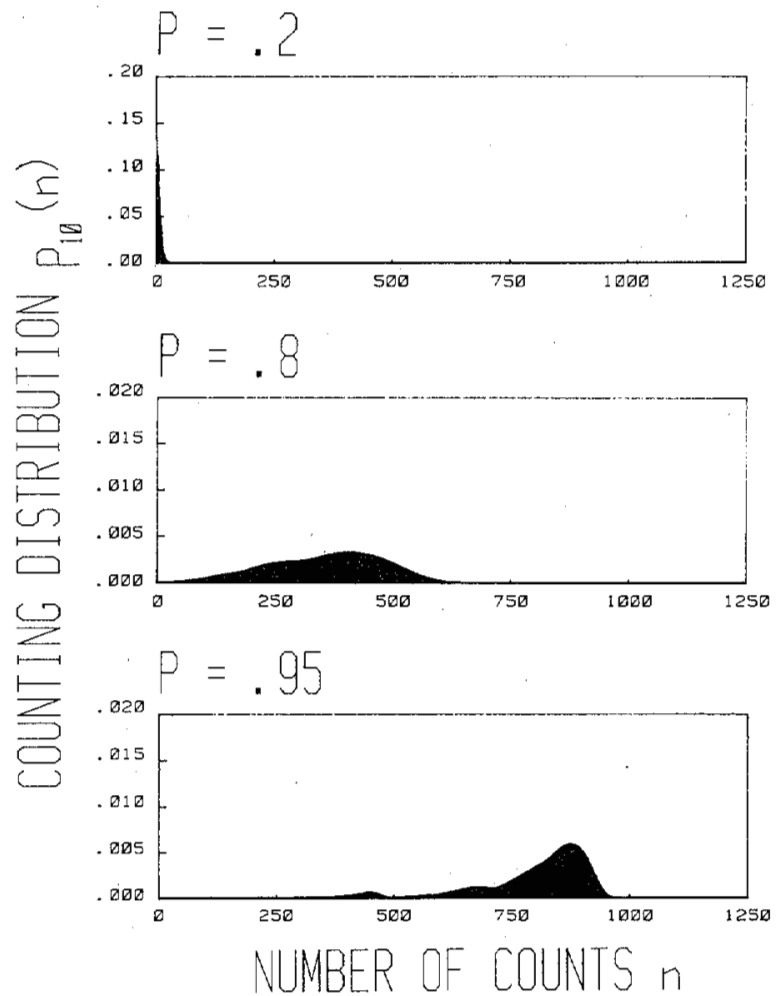

Fig. 5. Electron counting distribution $p_{10}(n)$ versus $n$, for $m=10$, with ionization probability $P=0.2$ (top), $P=0.8$ (middle), and $P=0.95$ (botiom). The maximum multiplication is $2^{10}=1024$.

multiply at the first conduction-band step in Fig. 1(b). It, plus all of its progeny, are confined to an ultimate gain that can be no larger than $(1+P)^{m-1}$. For the particular 
example of $P=0.95$ and $m=10$, the maximum gain is limited to $(1.95)^{9} \approx 400$. This is indeed the number of counts at which a first peak is evident in the bottom distribution in Fig. 5. The different peaks result from other such failures. Of course, as $P$ approaches unity, the multiple peaks disappear and the distribution narrows to a delta function (at 1024 counts in Fig. 5). Close inspection of Fig. 4 reveals that it, too, has multiple peaks.

\section{ImPULSE Response Function}

The time course of the current pulse produced in an external circuit by the SAPD may be determined from the motion of the carriers in the device. The current pulse arising from a single photon absorption is a superposition of contributions from the initial electron and from the electrons and holes created at the conduction-band steps. The physical basis of the impulse response function is most readily understood in terms of deterministic transit time. The transit times of the two carrier species (across a single graded region) are designated as $\tau_{e}$ and $\tau_{h}$, respectively (the net velocity of holes generally differs from that of electrons; we define $r=\tau_{h} / \tau_{e}$ ). Subsequently, the transit time is permitted to assume the form of a random variable. This provides a more realistic model for the impulse response function. Although the probability density function (or value) for the transit time can in general differ from stage to stage, it is convenient (and reasonable) to choose them to be independent identically distributed random variables. In Section IV, we provide graphical impulse response functions both when the transit times are deterministic and when they are distributed as (truncated) Gaussian probability density functions.

\section{A. Underlying Physical Basis}

An examination of Fig. 1(b) demonstrates that the initially injected electron drifts the entire length of the device before arriving at the $\mathrm{n}^{+}$contact. Assuming uniform electric-field and short-circuit device conditions, this produces a rectangular current pulse in the circuit. The duration of this contribution is $(m+1) \tau_{e}$, and its height is $q /(m+$ 1) $\tau_{e}$, so that the area is precisely the electronic charge $q$ [21]. The quantity $\tau_{e}$ is the time it takes the electron to drift across a single graded region of the superlattice; $(m+1) \tau_{e}$ is the time required to drift across the entire device. The initial hole does not contribute to the current because it does not traverse any distance.

If this initial electron creates an electron-hole pair at the first conduction-band step (this happens with probability $P$ ), the daughter electron gives rise to a current pulse in the circuit that is delayed by $\tau_{e}$ and of shorter duration than the mother pulse (i.e., $m \tau_{e}$ rather than $(m+1) \tau_{e}$ ), but of the same magnitude $\left(q /(m+1) \tau_{c}\right)$. The conjugate hole, on the other hand, travels backward traversing only one graded region before reaching the contact. It therefore contributes a rectangular current pulse delayed by $\tau_{e}$ and of duration $\tau_{h}$, with magnitude $q /(m+1) \tau_{h}$. For each electron-hole pair created in the device, the integrals of
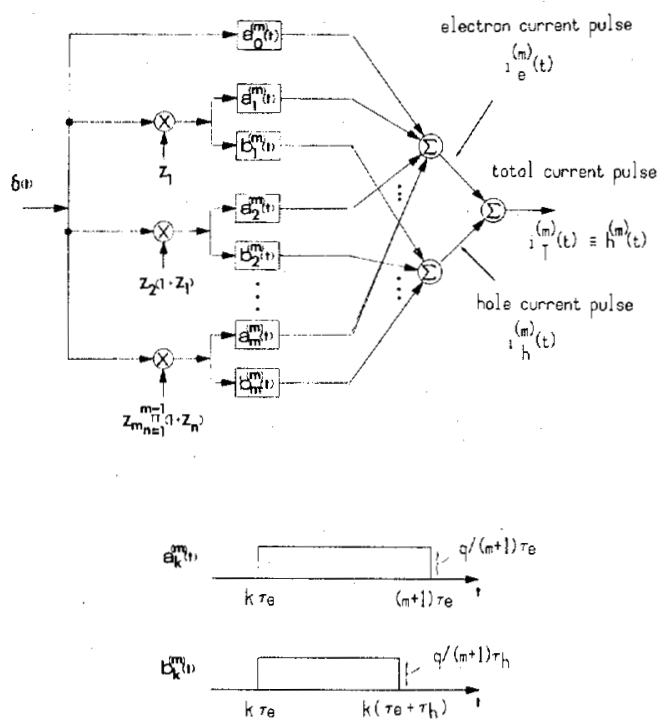

Fig. 6. Flow diagram detailing contributions to the single-photon impulse response function $h^{(m)}(t)$ for the $m$-stage SAPD with time dynamics. The transit times $\tau_{c}$ and $\tau_{h}$ are taken to be deterministic (as shown in the figure) or independent identically distributed Gaussian random variables. The input $\delta(t)$ represents the absorption of a single photon. The $a_{k}$ 's and $b_{k}$ 's are component electron and hole currents, respectively, whereas the $z$ 's are Bernoulli random variables.

the electron and hole currents produce a charge $q$ in the circuit [21].

The general case for the $m$-stage device is illustrated by means of the flow diagram of Fig. 6. The input $\delta(t)$ represents the absorption of a single photon. The current pulses produced by electrons and holes created at the $k$ th step are designated $a_{k}^{(m)}(t)$ and $b_{k}^{(m)}(t)$, respectively (see lower portion of Fig. 6). The symbols $z_{1}, z_{2}, \cdots, z_{m}$ represent independent identically distributed Bernoulli random variables such that $\operatorname{Pr}[z=0]=1-P$ and $\operatorname{Pr}[z=1]=P$. The electron current pulse $i_{e}^{(m)}(t)$ and the hole current pulse $i_{h}^{(m)}(t)$ are weighted sums of the $a_{k}$ 's and the $b_{k}$ 's, respectively. The total current pulse $i_{T}^{(m)}(t)$ is, in turn, a superposition of the electron and hole currents. Since the total current pulse is the response to a single primary event it is simply designated as $i(t)$, or as the single-photon impulse response function $h^{(m)}(t)$.

\section{B. Theory}

The flow diagram of Fig. 6 is easily translated into a mathematical expression for the (random) total current $i_{T}^{(m)}(t)=i(t)=h^{(m)}(t)$

$$
\begin{aligned}
i(t)=a_{0}^{(m)}(t) & +\sum_{k=1}^{m} z_{k}\left[a_{k}^{(m)}(t)+b_{k}^{(m)}(t)\right] \prod_{n=1}^{k-1}\left(1+z_{n}\right), \\
m & \geq 1 .
\end{aligned}
$$

The functions $a_{k}^{(m)}(t)$ and $b_{k}^{(m)}(t)$ are displayed in the lower portion of Fig. 6 .

The mean current is written as

$$
\begin{aligned}
\langle i(t)\rangle= & \left\langle a_{0}^{(m)}(t)\right\rangle+P \sum_{k=1}^{m}(1+P)^{k-1} \\
& \cdot\left[\left\langle a_{k}^{(m)}(t)\right\rangle+\left\langle b_{k}^{(m)}(t)\right\rangle\right] .
\end{aligned}
$$


Expressions for the mean-square current $\left\langle[i(t)]^{2}\right\rangle$ and the autocorrelation function of the current $R^{(m)}\left(t_{1}, t_{2}\right)$ are quite lengthy and therefore not included here.

In the general case of random transit time, the mean values of the quantities $a_{k}^{(m)}(t)$ and $b_{k}^{(m)}(t)$ are

$$
\begin{aligned}
& \left\langle a_{0}^{(m)}(t)\right\rangle=q \int_{t}^{\infty} \frac{1}{\alpha}{ }^{m+1} \circledast f(\alpha) d \alpha \\
& \left\langle a_{k}^{(m)}(t)\right\rangle=q \int_{0}^{t} \stackrel{m-k+1}{\circledast} f(\alpha) d \alpha \\
& \cdot \int_{t-\alpha}^{t} \frac{1}{\alpha+\beta} \stackrel{k}{\circledast} f(\beta) d \beta \\
& \left\langle b_{k}^{(m)}(t)\right\rangle=q r^{2} \int_{0}^{\infty} \stackrel{m-k+1}{\circledast} f(\alpha) d \alpha \\
& \cdot \int_{0}^{t} \frac{1}{\alpha+\beta} \circledast f(\beta) d \beta \int_{t-\beta}^{t} \circledast f_{0}(\gamma) d \gamma
\end{aligned}
$$

where the symbol $\stackrel{n}{\circledast}$ represents $n$-fold self-convolution.

The results presented above are expressed in terms of the density functions for the transit time $f(\tau)$. All transit times have been assumed to be independent and identically distributed. Results for the special case of deterministic transit are recovered by using the density function

$$
f\left(\tau_{e}\right)=\delta\left(\tau_{e}-\left\langle\tau_{e}\right\rangle\right)
$$

along with a similar expression for the holes. For the random case, the transit time density functions are assumed to be truncated Gaussian random variables of the form

$$
\begin{aligned}
f(\tau) & =\frac{c}{\left(2 \pi \sigma_{e}^{2}\right)^{1 / 2}} \exp \left[-\left(\tau-\tau_{e}\right)^{2} / 2 \sigma_{e}^{2}\right], & & \tau \geq 0 \\
& =0, & & \tau<0 .
\end{aligned}
$$

For simplicity, the parameters $\tau_{e}$ and $\sigma_{e}$ represent the mean and standard deviation of the Gaussian electron transit time per stage, respectively. An identical density function is used for the hole transit time, with mean value $\tau_{h}$. For the purposes of calculation we choose $\sigma_{e} / \tau_{e}=\sigma_{h} / \tau_{h}=0.2$, in accord with typical experimental results for the quaternary materials of interest in optical communications [22], [23]. The quantity $c$ is a normalization constant satisfying

$$
\int_{0}^{\infty} f(\tau) d \tau=1
$$

The purpose of the truncation is to disallow negative transit times.

\section{Results for the Time Response}

The time course of the output current from the SAPD, in response to a single photon absorption at the input, will be determined in large part by the transit time of the carriers in the device. It is useful to determine the dependence of the impulse response function on various parameters of the model in order to understand how the SAPD
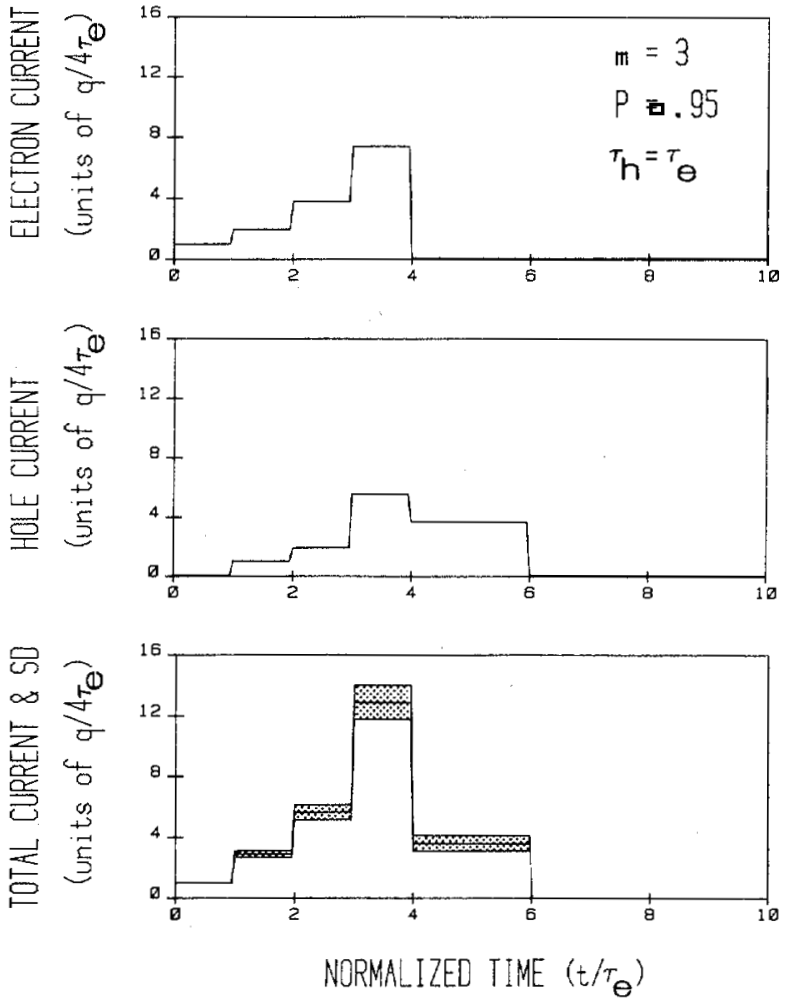

Fig. 7. Impulse response function for a three-stage SAPD with deterministic transit time, $r=\tau_{h} / \tau_{e}=1$, and $P=0.95$. The upper and middle portions of the figure represent the mean electron current and mean hole current, respectively. The lower portion of the figure displays the mean total current $i(t)=h^{(m)}(t)$, together with its one-standard deviation (SD) limits (stippled region). The ordinate represents current (in units of $q / 4 \tau_{e}$ ) whereas the abscissa represents normalized time (in units of $t / \tau_{e}$ ). The output current pulse extends over a time $6 r_{e}$ in response to a single photon absorption at $t=0$.

will perform in an optical system. For purposes of comparison, the examples provided in the following are all for a three-stage device. The role of the following parameters is studied: i) randomness in the transit time (results are presented for deterministic and for Gaussian transit time); ii) the impact ionization probability $P$ (results are presented for $P=0.95$ and $P=0.8$ ); and iii) the relative transit times of holes and electrons $r=\tau_{h} / \tau_{e}$ (results are presented for $r=1$ and $r=2$ ). Impulse response functions have been calculated for many other values of $m, P$, and $r$ the results provided here are representative. Deterministic transit time is considered first.

\section{A. Deterministic Transit Time}

In Fig. 7 we present the impulse response function for an $m=3$ device with $r=1$ and $P=0.95$. The upper and middle portions of the figure represent the mean electron current and mean hole current, respectively. The lower portion of the figure displays the mean total current $i(t)$, together with the one-standard deviation (SD) limits (stippled region). The results are calculated from (13) and (14). The ordinate represents current (in units of $q / 4 \tau_{e}$ ) whereas the abscissa represents normalized time (in units of $t / \tau_{\ell}$ ). The contribution to the external current arising from electrons in the device extends to $(m+1) \tau_{e}$ whereas the hole 

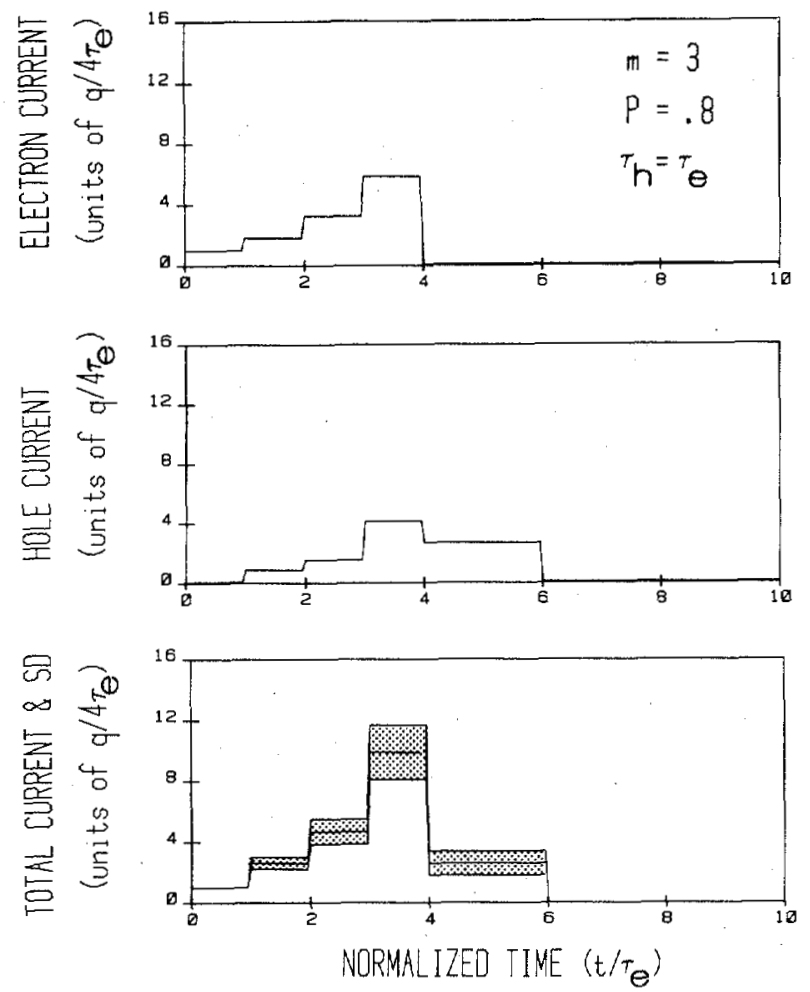

Fig. 8. Impulse response function for a three-stage SAPD with deterministic transit time $r=1$ and $P=0.8$. A comparison of this result with that provided in Fig. 7 demonstrates that the average charge induced in the circuit is reduced while the standard deviation of the average total current is increased. Again the output current pulse extends over a time $6 \tau_{a}$ in response to a single photon absorption at $t=0$.

contribution extends a longer time, to $m\left(\tau_{e}+\tau_{h}\right)$, as indicated earlier. The angular form of the total average current results from the assumptions of deterministic transit time and integer $r$. The area under the total average current is, of course, given by $(1+0.95)^{m} \approx 7.41$.

In Fig. 8 we show the impulse response function when all parameters are the same as in Fig. 7 except the impact ionization probability $P$, which is taken to be $P=0.8$. The lower value of $P$ results in a smaller total charge induced in the circuit $\left(1.8^{3} \approx 5.83\right.$ instead of 7.41 as above). It also results in an increased standard deviation of the average total current; this arises from the additional uncertainty in the carrier generation process.

The effects of a decreased hole drift velocity (by a factor of 2 relative to the electron drift velocity) are illustrated in Figs. 9 and 10. These impulse response functions are analogous to those presented in Figs. 7 and 8 ( $m=3$; $P=0.95,0.8$ ) except that here $r=2$ instead of 1 . The current contribution arising from electrons (upper portion of figures) is independent of $r$. The slower hole contributions, on the other hand, cause the duration of the total current pulse to increase to $3\left(\tau_{e}+2 \tau_{e}\right)=9 \tau_{e}$. The role of $P$ is the same as that seen above; decreasing $P$ reduces the area (which is independent of $r$ ) and increases the standard deviation of the total current pulse.

\section{B. Random Transit Time}

Finally, in Fig. 11, we present the impulse response function when the transit time is Gaussian rather than deterministic. The coefficients of variation of the transit time
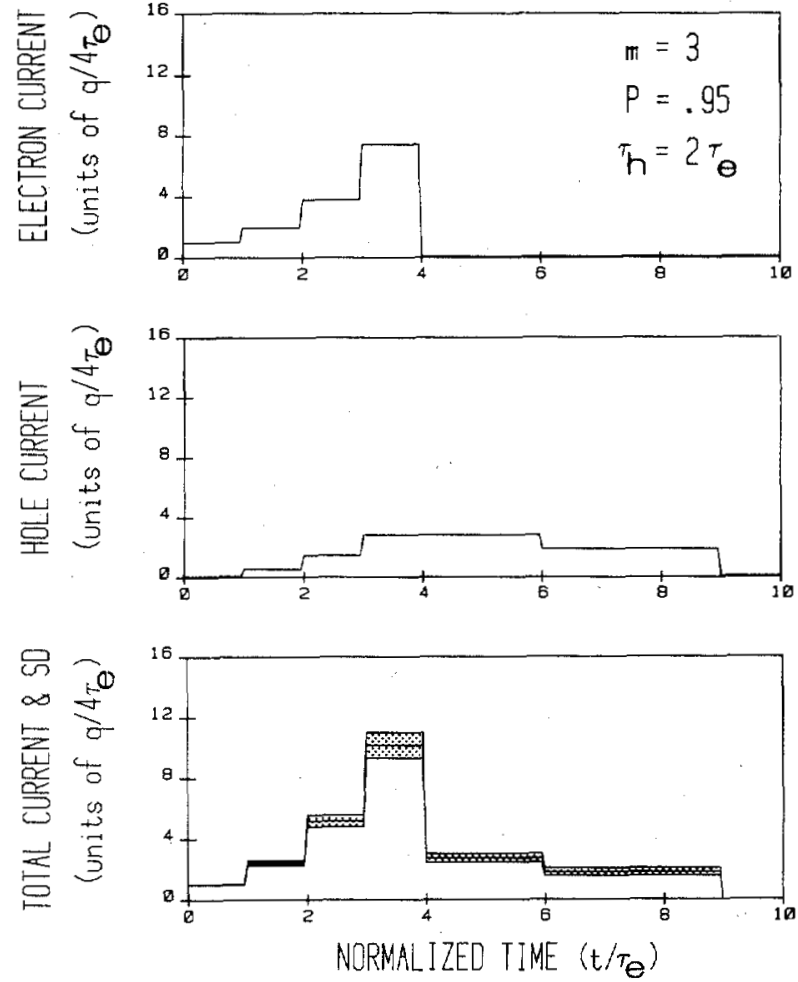

Fig. 9. Impulse response function for a three-stage SAPD with deterministic transit time $r=2$ and $P=0.95$. The contribution to the current arising from electrons (upper portion of figure) is the same as that shown in Fig. 7. The slower hole contribution causes the total output current pulse to extend over a time $9 \tau_{e}$ in response to a single pheton absorption at $t=0$.
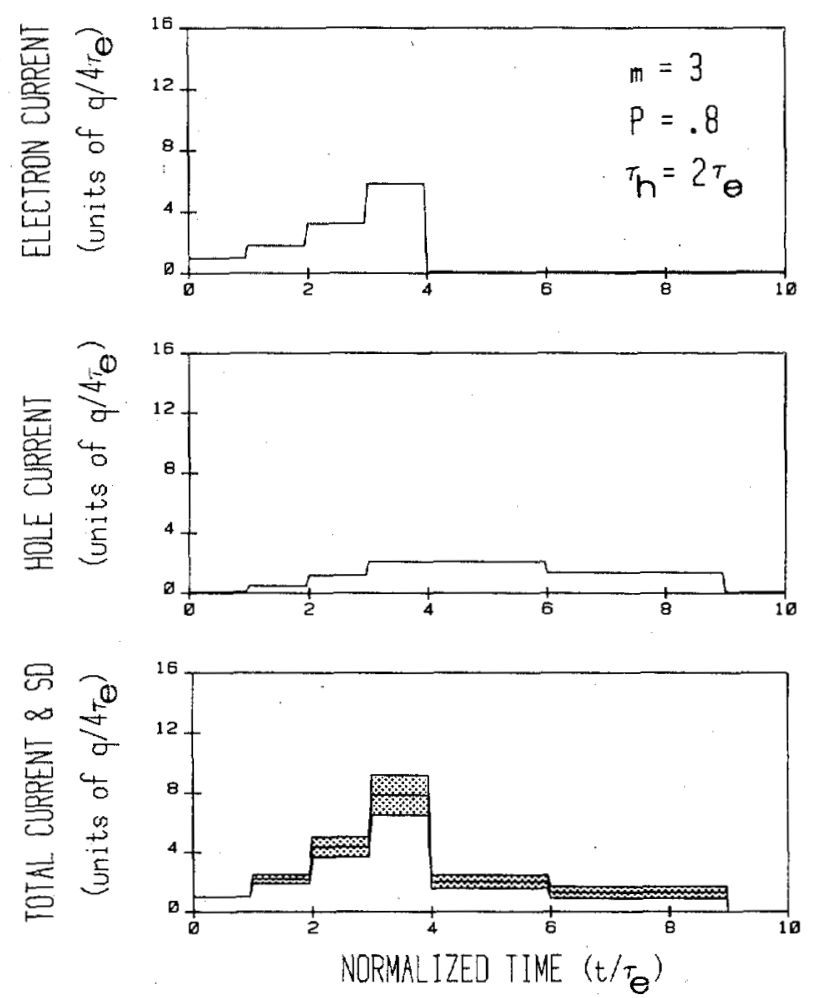

Fig. 10. Impulse response function for a three-stage SAPD with determin istic transit time $r=2$ and $P=0.8$. A comparison of this result with that provided in Fig. 9 demonstrates that the average charge induced in the circuit is reduced while the standard deviation of the total average current is increased. Again the output current pulse extends over a time $9 \tau_{e}$ in response to a single photon absorption at $t=0$. 

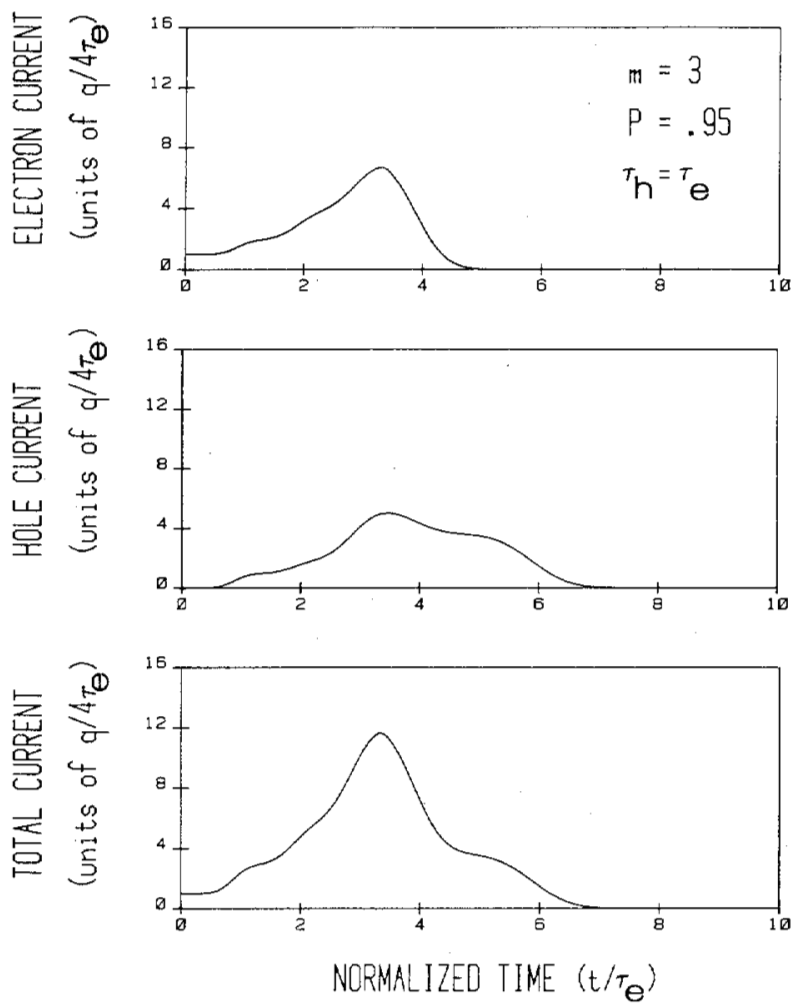

Fig. 11. Impulse response function for a three-stage SAPD with Gaussian transit time $\left(\sigma_{e} / \tau_{\mu}=\sigma_{h} / \tau_{h}=0.2\right), r=1$, and $P=0.95$. The parameters and presentation are identical with those used in Fig. 7. Comparing the results iliustrates that randomness in the transit time imparts substantial smoothing to the impulse response function. The current pulse (in response to a single photon absorption at $t=0$ ) extends beyond $6 \tau$, and has a tail, as expected. Taking account of the electron-injection dispersion would smooth the initial portion of the impulse response function. The result portrayed here is more representative of what might be expected from a real device than is the idealized result in Fig. 7.

$\left(\sigma_{e} / \tau_{e}\right.$ and $\left.\sigma_{h} / \tau_{h}\right)$ are chosen to be 0.2 in accordance with expectations for quaternary materials [22], [23]. The parameters $(m=3, r=1, P=0.95)$ and presentation are otherwise identical to those of Fig. 7.

A comparison shows that the spread of carrier travel time has the effect of smoothing and extending the impulse response function. Behavior of this kind is obtained for the entire range of parameter values studied. The flat initial portion of the impulse response function (which has an approximate duration $\tau_{e}$ ) results from the assumption of delta-function photon absorption at the $\mathrm{p}^{+}$boundary in the device (see Fig. 1(b)). In a real device, photon absorption will take place more gradually, introducing a dispersion into the initial electron injection. The principal consequence of this effect, which can be readily calculated, will be to remove the flatness from the initial portion of the impulse response function shown in Fig. 11. Various kinds of specially designed absorption regions can be introduced prior to the staircase in order to optimize absorption [24]. This will permit greater multiplication to take place in the device, but at the expense of a decrease in the time-bandwidth product. The time response portrayed here for the random-transit-time case is clearly more representative of what might be expected for a real device than is the idealized time response displayed in Fig. 7.

\section{CONCLUSION}

A mathematical model describing the multiplication noise and time response of the staircase avalanche photodiode has been developed. Valence-band discontinuities are assumed to be sufficiently small so that holes do not impact-ionize. For instantaneous multiplication, the excess noise factor and carrier counting distributions were calculated and displayed graphically for various values of the number of stages $m$ and the impact ionization probability $P$. Single-photon impulse response functions were obtained for both deterministic and random transit times for several values of $m, P$, and $r$ (the ratio of hole and electron transit times)

The impulse response function was found to have a full width given by $\tau_{p}=m\left(\tau_{e}+\tau_{h}\right)$, under the assumption that the carriers undergo deterministic transit time. Under the more realistic assumption of random time delay, the half width will be somewhat smaller than this. The theoretical gain-bandwidth product is given approximately by $G B \approx(1+P)^{m} / m\left(\tau_{e}+\tau_{h}\right)$. Numerical estimates for $\tau_{p}$ and $G B$ expected for a typical quarternary detector (e.g., $\mathrm{AlGaAsSb} / \mathrm{GaSb}$ on GaSb substrate) designed for the 1.31.6- $\mu \mathrm{m}$ wavelength low-loss-fiber region can be calculated. Choosing typical hole and electron velocities $v_{h} \approx$ $5 \times 10^{6} \mathrm{~cm} / \mathrm{s}$ and $v_{e} \approx 1 \times 10^{7} \mathrm{~cm} / \mathrm{s}$, respectively, and a device layer thickness of $3000 \AA$, yields hole and electron current contribution with durations of 6 and $3 \mathrm{ps} / \mathrm{stage}$, respectively. For a five-stage device, $\tau_{p} \approx 45 \mathrm{ps}$, corresponding to a bit rate of about $22 \mathrm{Gbit} / \mathrm{s}$. The finite absorption length of the light will alter $\tau_{p}$ slightly. Other effects will further limit the maximum bit rate. The gainbandwidth product $G B \approx 600 \mathrm{GHz}$, assuming a five-stage device with $P=0.95$, which has a gain $\approx 28$.

From an experimental point of view, the first attempts are under way at AT\&T Bell Laboratories to construct a staircase device using molecular-beam epitaxy [24]. From a theoretical point of view, quite a bit of additional work needs to be done to fully analyze the behavior of the SAPD. The emphasis in this paper has been on the time response of the device and on multiplication noise arising from the branching process; calculations for additive nonmultiplying and multiplying dark noise should also be carried out. The effects of residual hole impact ionization, and the possibility of hole trapping, should be considered. The filtering of the time signal by various processes (e.g., the circuit time constant) should be incorporated into the model. Studies of system performance, including error probabilities and intersymbol interference, are also of interest. The first step along these lines, recently carried out [25], is the calculation of counting distributions and bit error rates for SAPD devices illuminated by Poisson light pulses (e.g., those from a laser) rather than by single photons. Brennan [26] has recently conducted a simulation study of electron and hole impact ionization in the SAPD. No particular difficulties are expected in any of these endeavors, but it is important to explore them so that an effective comparison can be drawn between system performance expected with staircase and conventional APD devices. 


\section{ACKNOWLEDGMENT}

The authors are indebted to F. Capasso of AT\&T Bell Laboratories, R. J. McIntyre of RCA Laboratories, and M. Littlejohn of North Carolina State University for many valuable suggestions.

\section{REFERENCES}

[1] J. M. Senior, Optical Fiber Communications. Englewood Cliffs, NJ: Prentice-Hall, 1985

[2] R. J. McIntyre, "Multiplication noise in uniform avalanche diodes," IEEE Trans. Electron Devices, vol. ED-13, pp. 164-168, 1966.

[3] —, "The distribution of gains in uniformly multiplying avalanche photodiodes: Theory," IEEE Trans. Electron Devices, vol. ED-19, pp. 703-713, 1972.

[4] P. P. Webb, R. J. McIntyre, and J. Conradi, "Properties of avalanche photodiodes," RCA Rev., vol. 35, pp. 234-278, 1974.

[5] S. D. Personick, "New results on avalanche multiplication statistics with application to optical detection," Bell Syst. Tech.J., vol. 50, pp. $167-189,1971$

[6] S. D. Personick, "Statistics of a general class of avalanche detectors with applications to optical communication," Bell Syst. Tech. J., vol. 50, pp. 3075-3095, 1971.

[7] K. Matsuo, M. C. Teich, and B. E. A. Saleh, "Poisson branching point processes," J. Math. Phys., vol. 25, pp. 2174-2185, 1984.

[8] G. U. Yule, "A mathematical theory of evolution, based on the conclusions of Dr. J. C. Willis, F. R. S.," Phil. Trans. Royal Soc. London, vol. B213, pp. 21-87, 1924

[9] W. Furry, "On fluctuation phenomena in the passage of high energy electrons through lead," Phys. Rev., vol. 52, pp. 569-581, 1937.

[10] G. E. Stillman, V. M. Robbins, and N. Tabatabaie, "III-V compound semiconductor devices: Optical detectors," IEEE Trans. Electron. Devices, vol. ED-31, pp. 1643-1655, 1984.

[11] R. Chin, N. Holonyak, G. E. Stillman, J. Y. Tang, and K. Hess, "Impact ionization in multilayered heterojunction structures," Electron. Lett., vol. 16, pp. 467-469, 1980.

[12] F. Capasso, W. T. Tsang, A. L. Hutchinson, and G. F. Williams "Enhancement of electron impact ionization in a superlattice: A new avalanche photodiode with a large ionization rate ratio," Appl. Phys. Lett., vol. 40, pp. 38-40, 1982.

[13] G. F. Williams, F. Capasso, and W. T. Tsang, "The graded bandgap multilayer avalanche photodiode: A new low-noise detector," IEEE Electron. Device Lett., vol. EDL-3, pp. 71-73, 1982.

[14] F. Capasso, W. T. Tsang, and G. F. Williams, "Staircase solid-state photomultipliers and avalanche photodiodes with enhanced ionization rates ratio," IEEE Trans. Electron Devices, ED-30, pp. 381-390, 1983.

[15] F. Capasso, "Band-gap engineering via graded gap, superlattice, and periodic doping structures: Applications to novel photodetectors and other devices," J. Vac. Sci. Tech. B, ser. 2, vol. 1, pp. 457-461, 1983.

[16] G. E. Stillman and C. M. Wolfe, "Avalanche photodiodes," in Semiconductors and Semimetals, vol. 12, Infrared Detectors II, R. K. Willardson and A. C. Beer, Eds. New York: Academic, 1977, pp. 291394.

[17] T. E. Harris, The Theory of Branching Processes. New York: Springer, 1963.

[18] K. M. van Vliet and L. M. Rucker, "Theory of carrier multiplication and noise in avalanche devices-Part I: One-carrier processes," IEEE Trans. Electron Devices, vol. ED-26, pp. 746-751, 1979.

[19] K. M. van Vliet, A. Friedmann, and L. M. Rucker, "Theory of carrier multiplication and noise in avalanche devices-Part II: Two-carrier processes," IEEE Trans. Electron Devices, vol. ED-26, pp. 752$764,1979$.

[20] B. E. A. Saleh, Photoelectron Statistics. New York: Springer, 1978

[21] R. J. McIntyre, RCA Electro-Optics Photodetectors, Ste. Anne de Bellevue, Quebéc, Canada, personal communication.

[22] F. Capasso, AT\&T Bell Laboratories, Murray Hill, NJ, personal communication.

[23] M. Littlejohn; North Carolina State University, personal communication

[24] F. Capasso, AT\&T Bell Laboratories, Murray Hill, NJ, personal communication.

[25] K. Matsuo, M. C. Teich, and B. E. A. Saleh, "Performance of fiberoptic communication systems using the staircase avalanche photodiode," submitted for publication.

[26] K. Brennan, "Theory of electron and hole impact ionization in quantum well and staircase superlattice avalanche photodiode structures," IEEE Trans. Electron Devices, vol. ED-32, pp. 2197-2215, Nov. 1985.

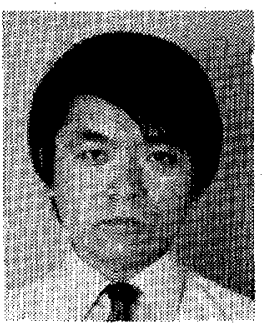

Kuniaki Matsuo was born in Hiroshima, Japan. He received the B.S. degree in electrical engineering from Tokyo Electrical Engineering Colege, Tokyo, in 1972 and the M.S. and Ph.D. degrees in electrical engineering from Columbia University, New York, NY, in 1978 and 1984, respectively.

$\mathrm{He}$ is presently a Postdoctoral Research Scientist in the Columbia Radiation Laboratory, Department of Electrical Engineering, Columbia University, where he is pursuing his research interests in avalanche photodetection, quantum electronics, and point processes.

Dr. Matsuo is a member of Sigma Xi.

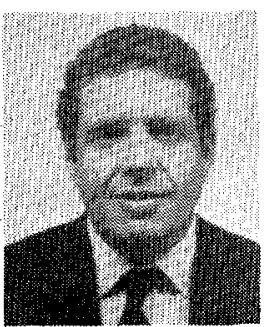

Malvin C. Teich (S'62-M'66-SM'72) was born in New York, NY, on May 4, 1939. He received the S.B. degree in physics from the Massachusetts Institute of Technology, Cambridge, in 1961, the M.S. degree in electrical engineering from Stanford University, Stanford, CA, in 1962, and the $\mathrm{Ph} . \mathrm{D}$. degree in quantum electronics from Cornell University, Ithaca, NY, in 1966.

In 1966 he joined the MIT Lincoln Laboratory, Lexington, MA, where he was engaged in work on coherent detection and modulation. In 1967, he became a member of the faculty in the Department of Electrical Engineering, Columbia University, New York, NY, where he is now teaching and pursuing his research interests in the areas of optical and infrared detection, quantum optics, lightwave comminications, and sensory perception He served as Chairman of the Department from 1978 to 1980 . He is also a member of the faculty in the Department of Applied Physics and Nuclear Engineering, and a member of the Columbia Radiation Laboratory, the Center for Telecommunications Research, and the Columbia Bioengineering Institute. He has authored or coauthored over 100 technical publications, and holds one patent.

Dr. Teich is a member of Sigma Xi, the American Physical Society, the Acoustical Society of America, the Society for Neuroscience, and the New York Academy of Sciences. He served as a member of the Editorial Advisory Panel for Optics Letters from 1977 to 1979. In 1969 he was the recipient of the IEEE Browder J. Thompson Memorial Prize Award for his paper "Infrared Heterodyne Detection," and in 1973 he was appointed a Fellow of the John Simon Guggenheim Memorial Foundation. He received the $\mathrm{Ci}$ tation Classic Award of Current Contents in 1981 and was elected a Fellow of the Optical Society of America in 1983.

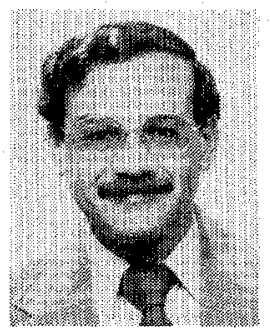

Bahaa E. A. Saleh (M'73) received the B.S. degree from Cairo University in 1966 and the Ph.D. degree from the Johns Hopkins University in 1971 , both in electrical engineering.

From 1971 to 1974 he was an Assistant Professor at the University of Santa Catarina, Brazil. Thereafter, he joined the Max Planck Institute in Göttingen, Germany, where he was involved in research in laser light scattering and photon correlation spectroscopy. He is presently Professor of Electrical and Computer Engineering at the University of Wisconsin, Madison, where he has been since 1977. He held visiting appointments at the University of Kuwait in.1976, the School of Optometry of the University of California, Berkeley, in 1977, and the Columbia Radiation Laboratory of Columbia University in 1983. He is currently involved in research in image processing, optical information processing, statistical optics, optical communication, and vision. He is the author of Photoelectron Statistics (Springer, 1978) and a co-editor of Transformations in Optical Signal Processing (SPIE, 1981). In 1980-1983 he was an associate editor of the Journal of the Optical Society of America, and since 1983 he has been a topical editor of the same journal.

Dr. Saleh is a Fellow of the Optical Society of America and a member of Phi Beta Kappa and Sigma Xi. He was one of the recipients of the 19841985 Guggenheim Fellowship. 